\begin{tabular}{lll} 
Pădagogik Aktuelle Forschungs- und Medienprojekte \\
\cline { 2 - 4 }
\end{tabular}

Der Deutsche Jugendvideopreis 2013 und 2014 - Analyse der Zusammensetzung der Teilnehmenden unter besonderer Berücksichtigung des Geschlechts

\section{LARISSA SCHMIEDE}

\section{Relevanz von Filmwettbewerben für Heranwachsende}

Kinder und Jugendliche sehen die Welt mit anderen Augen - daher muss ihnen die Möglichkeit eröffnet werden, ihre eigenen Sichtweisen und Interessen auszudrücken. Hierfür bietet ihnen Film als audiovisuelles Medium vielfache Gestaltungsmöglichkeiten. Durch eigene Filmproduktionen können Heranwachsende ihre Ausdrucksformen zur Artikulation der persönlichen Lebenswelt erweitern. Filmwettbewerbe wiederum bieten den jungen Produzent/-innen die Chance, ihre Filme und damit ihre Interessen einer breiten Öffentlichkeit zu präsentieren. $\mathrm{Ob}$ diese Möglichkeit in gleichem Maße von Mädchen und Jungen genutzt wird, wurde im Rahmen einer Bachelorthesis des Studiengangs Kultur- und Medienbildung untersucht. Denn nur durch eine ausgewogene Teilnahme beider Geschlechter können die Interessen und Sichtweisen von weiblichen und männlichen Heranwachsenden zur Geltung gebracht werden.

Die Arbeit basiert auf der Annahme, dass der Grund für mögliche geschlechtsspezifische Differenzen der jeweiligen Fähigkeiten und individuellen Entwicklungsmöglichkeiten nicht in der biologischen Determination von Geschlecht liegt, sondern in seiner sozialen Konstruktion (vgl. Butler 1991, S. 39). Filmarbeit hat ein hohes Potenzial für die Auflösung stereotyper Geschlechterrollen, da sie Heranwachsende mittels der Auseinandersetzung mit medialen (Geschlechter-) Bildern in ihrer Medien-, aber auch in ihrer Genderkompetenz fördert (vgl. DeckeCornill/Luca 2010, S. 11). Die meisten in der Medienpädagogik formulierten Medienkompetenzmodelle nehmen jedoch keinen expliziten Bezug auf die Genderproblematik und in den Konzepten zur Genderkompetenz bleibt wiederum der Umgang mit Medien weitgehend unberücksichtigt. Eine Verknüpfung dieser beiden Themenfelder wird im Begriff der geschlechtersensiblen Medienkompetenz nach Luca und Aufenanger (2007) deutlich, der für die Arbeit eine zentrale Bedeutung hat. Der Begriff wird dabei als Abgrenzung bzw. Erweiterung einer geschlechtsspezifischen Medienkompetenzförderung definiert, die sich auf eine bipolare Vorstellung von Zweigeschlechtlichkeit beschränkt.

Auf der Abwesenheit einer geschlechtsneutralen Wirklichkeit beruhen auch die Bemühungen zur Gleichstellung der Geschlechter, die zum Ziel haben, dass beiden Geschlechtern in gleichem Maße persönliche Entwicklungsmöglichkeiten zur Verfügung stehen. Dieser Maxime entsprechend ist die Aufgabe von Filmwettbewerben für Heranwachsende, die Freiheit und den gesellschaftlichen Raum zu schaffen, den diese zur geschlechtsunabhängigen Realisation ihrer individuellen Lebensentwürfe benötigen. Um zu überprüfen, ob dies gelingt, wurden in der Bachelorthesis exemplarisch die Teilnehmenden des Deutschen Jugendvideopreises (DJVP) untersucht. Der Wettbewerb wird jährlich vom Kinder- und Jugendfilmzentrum in Deutschland (KJF) durchgeführt und richtet sich an weibliche und männliche Heranwachsende unter 26 Jahren. Da er zudem keine Vorgaben zu Produktionsart, Länge der Filme und Themenwahl macht, eignet er sich besonders gut als Untersuchungsfeld. Ferner hat die Verfasserin der Arbeit als Mitglied des Auswahlgremiums einen erleichterten Zugang zum Forschungsfeld.

Das Gebiet der Filmwettbewerbe ist bisher weitestgehend unerforscht. Sie spielen jedoch eine große Rolle für die Förderung von Kindern und Jugendlichen, da sie ihre Interessen und Sichtweisen ernst nehmen und deren Ausdruck im Medium Film unterstützen. Die filmischen Eigenproduktionen Heranwachsender bieten der Wissenschaft zudem authentische Einblicke in deren Lebenswelten (vgl. Barg/ Niesyto/ Schmolling 2006). Dieses selten genutzte 
Potential wurde durch eine Untersuchung des Entstehungskontexts und des Einflusses von Geschlecht auf die aktive Auseinandersetzung mit Film aufgegriffen. Zur umfassenden Klärung der zentralen Forschungsfrage nach der Geschlechterverteilung unter den Teilnehmenden des Deutschen Jugendvideopreises wurden dabei auch Ursachen und Gründe der Verteilung und Möglichkeiten, diese zu beeinflussen, ermittelt.

\section{Methodik}

Dazu fand eine Verknüpfung der quantitativen mit der qualitativen Forschung statt, indem zuerst eine quantitative Auswertung der Teilnahmebögen hinsichtlich der Teilnehmenden und Preisträger/-innen sowie der Produktionsarten im Hinblick auf Altersgruppe und Geschlecht vorgenommen wurde. Anschließend wurden Interviews mit vier Experten geführt, die innerhalb des Wettbewerbs wichtige Funktionen wie die Organisation oder das Sichten der Filme übernehmen und daher besondere Einblicke sowie spezielle Kenntnisse besitzen. Durch diese Kombination der Methoden konnte der Forschungsgegenstand aus unterschiedlichen Perspektiven betrachtet werden.

Für die Arbeit hatten das Geschlecht des Regisseurs und sein Geburtsdatum höchste Priorität. Da die Kategorie „Regisseur“ erst seit 2013 im Teilnahmebogen vorgesehen ist, wurden lediglich dieses und das Folgejahr berücksichtigt. Zudem wurden ausschließlich die Einreichungen der Altersgruppen B (11 15 Jahre) und C (16 - 20 Jahre) untersucht, da sich unter ihnen sowohl autonome als auch angeleitete Produktionen finden und die Altersspanne eine für die Filmbildung relevante Zielgruppe kennzeichnet

\section{Zentrale Befunde}

\section{Zusammensetzung der Teilnehmer- und Preisträ- ger/-innen}

Sowohl für 2013 als auch für 2014 wurde eine höhere Anzahl an Filmen männlicher Regisseure belegt und durch die Aussagen der Experten bestätigt (Abb. 1).

Ob die Befragten die ungleiche Geschlechterverteilung unter den Filmemachenden speziell in einer Altersgruppe, bei allen Teilnehmenden oder nur bei den Preisträgern bemerken, variiert allerdings. Alle Experten sind sich indes einig, dass die männlichen Filmemacher schon vor dem Jahr 2013 in der Überzahl waren - drei von ihnen bemerken jedoch in den letzten Jahren eine Zunahme weiblicher Teilnehmer. Dies bestätigt die quantitative Auswertung für Gruppe B, da 2014 im Gegensatz zum Vorjahr eine nahezu gleiche Anzahl weiblicher und männlicher Regisseure teilgenommen hat. In Anbetracht dessen, dass lediglich zwei Jahrgänge miteinander verglichen werden konnten, ist die Diagnose eines Trends jedoch nicht möglich.

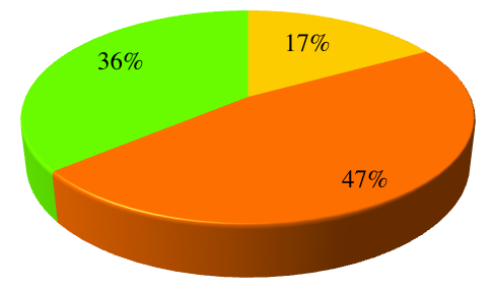

\section{ALTERSGRUPPE B}

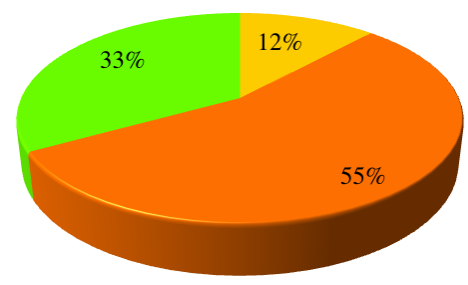

ALTERSGRUPPE C

$$
\begin{aligned}
& { }^{\square} \text { Gemischt } \\
& { }^{\square} \text { Männlich } \\
& { }^{\Xi} \text { Weiblich }
\end{aligned}
$$

Abb. 1: Geschlechterzusammensetzung der an dem Filmwettbewerb teilnehmenden Regisseur/-innen in den Altersgruppen B und C (2013 \& 2014).

Auch unter den Preisträger/-innen sind die Regisseurinnen in der Minderheit - besonders in der Altersgruppe B wird dies deutlich. Eine Ausnahme bildet im Jahr 2014 die Altersgruppe $C$ mit einem ausgewogenen Verhältnis prämierter Filme männlicher und weiblicher Regisseure. Besonders für dieses Jahr lässt sich gegenüber der Repräsentanz unter den Teilnehmenden keine Bevorzugung eines Geschlechtes bei der Prämierung erkennen. Der Aussage eines Experten folgend, kann ein Grund die für 2014 explizit formulierte Aufforderung des KJFs zur Förderung der weiblichen Teilnehmenden sein. Laut der Befragten liegt das Hauptaugenmerk bei der Auswahl und Prämierung jedoch auf der Qualität der Filme und nicht auf dem Geschlecht der Macher/-innen.

Die Gründe für die geringere Teilnahme weiblicher Regisseure konnten nicht abschlieBend geklärt werden. Die wissenschaftlichen Erkenntnisse und die Aussagen der Experten lassen jedoch vermuten, dass die negative Erwartungshaltung gegenüber ihrer Technikkompetenz dazu führt, dass sich Mädchen und junge Frauen weniger für die aktive Auseinandersetzung mit Film interessieren. Die aktive Medienarbeit kann dazu beitragen, diesen Vorurteilen entgegenzuwirken, um 
weiblichen Heranwachsenden ein breites Ausdrucks- und Entfaltungsspektrum zu präsentieren - auch im Hinblick auf eine spätere Berufswahl. Hinzu kommt die geringe Anzahl weiblicher Regisseure, die den jungen Filmemacherinnen als Vorbild dienen können. Die im Mediensystem dargestellten Identifikationsangebote helfen Heranwachsenden jedoch bei der Verortung im System der Zweigeschlechtlichkeit (Theunert 2005, S. 12), das im individuellen und kollektiven Bewusstsein der Menschen weiterhin vorhanden ist. Mangelt es in einer Berufsgruppe an Männlichkeits- und Weiblichkeitsentwürfen, kann dies einer freien Persönlichkeitsentwicklung entgegenwirken.

\section{Produktionsarten}

In beiden Jahren handelt es sich bei nahezu der Hälfte der Filme in Gruppe B um angeleitete Produktionen. In der Gruppe $\mathrm{C}$ ist die Anzahl dieser Produktionsart 2014 zwar im Vergleich zum Vorjahr signifikant gestiegen, trotzdem wurde nur ein Drittel der Filme mit professioneller Unterstützung produziert (Abb. 2).

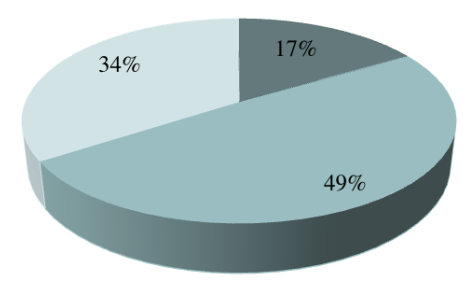

ALTERSGRUPPE B

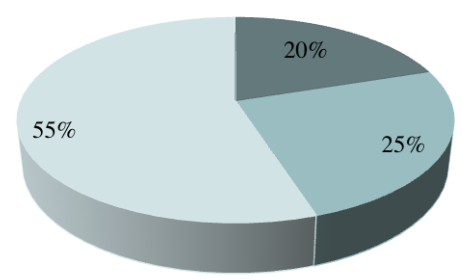

ALTERSGRUPPE C

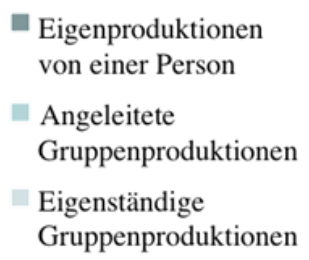

Abb. 2: Verteilung der Produktionsarten in den beiden Altersgruppen (2013 \& 2014).

Bei der Auswertung zeigte sich, dass die Einreichungen häufig im Schulkontext entstanden - in Film- und Medien-Arbeitsgemeinschaften oder im Rahmen des Informatik-, Kunst- und Filmunterrichts. Außerdem un- terstützten außerschulische Institutionen wie Filmcamps und -clubs oder Jugendhäuser die Heranwachsenden bei der Produktion. Die Filme der Gruppe $\mathrm{C}$ wurden meist eigenständig, also ohne pädagogische Hilfe, produziert. Sowohl in Altersgruppe B als auch in C wurden durchschnittlich nur $19 \%$ der Filme von ausschließlich einer Person angefertigt. Die Mehrzahl der Filme entsteht also in Teams - laut eines Experten fühlen sich Mädchen jedoch auf Grund mangelnden Selbstvertrauens und fehlender Akzeptanz oft nicht zur Führung einer Gruppe bereit. Auch in der wissenschaftlichen Literatur ist von einem Dominanzverhalten männlicher Heranwachsender innerhalb von gemischtgeschlechtlichen Gruppen die Rede, worin sich ein weiterer Grund für die geringe Anzahl an Produktionen weiblicher Filmemacher vermuten lässt (vgl. Buchen/Straub 2006, S. 97; Stolzenburg 1993, S. 136f.).

Die folgende Abbildung 3 zeigt die Verteilung der Produktionsarten unter weiblichen und männlichen Teilnehmenden.

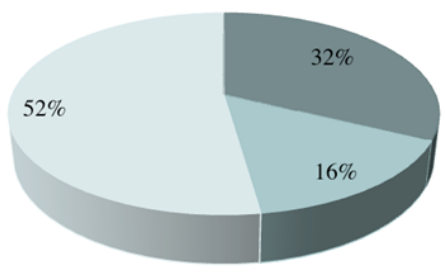

MÄNNLICHE TEILNEHMER B \& C

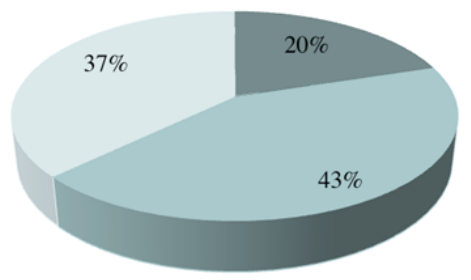

WEIBLICHE TEILNEHMER B \& C

Abb. 3: Verteilung der Produktionsarten bei männlichen und weiblichen Teilnehmern (2013 \& 2014).

Auffällig ist dabei die deutliche Überzahl der weiblichen Teilnehmer unter den angeleiteten Gruppenproduktionen in beiden Altersgruppen. Daraus kann auf ein größeres Angebot an aktiver Filmarbeit für Mädchen und junge Frauen geschlossen werden oder darauf, dass diese eher an solchen Projekten teilnehmen als die männlichen Heranwachsenden. In jedem Fall belegt die quantitative Auswertung die Funktion des DJVPs als Impulsgeber der filmpädagogischen Praxis besonders im Hinblick auf die Zielgruppe 
der weiblichen Heranwachsenden. Es steht zu vermuten, dass diese ohne professionelle Hilfe weitaus weniger Filme produzieren beziehungsweise beim DJVP einreichen würden die Unterrepräsentanz weiblicher Regisseure fiele dann möglicherweise noch deutlicher aus. Auch die Experten sehen in medienpädagogischen Projekten ein hohes Potenzial, der Ungleichverteilung der Geschlechter innerhalb des Wettbewerbs entgegenzuwirken. Eine geschlechtsheterogene oder -homogene Gruppenform wird laut der Experten und nach von Hören vom Medienprojekt Wuppertal e.V. (Interview in Luca/Aufenanger 2007, S. 136) jedoch vom inhaltlichen Gegenstand bestimmt und nicht andersherum und sollte jeweils nur phasenweise Anwendung finden, um das diskursive Konstrukt der Zweigeschlechtlichkeit (vgl. Butler 1991, S. 39) nicht zu reinszenieren. In jedem Fall kann eine Förderung der Medien- und Genderkompetenz durch die aktive Filmarbeit nur unter Einbezug aller Aspekte der geschlechtersensiblen medienpädagogischen Bildungsarbeit stattfinden.

Die Experten beobachten außerdem, dass die Filmemacher/-innen immer häufiger Themen wählen, die tradiert mit dem jeweils anderen Geschlecht assoziiert werden. Dieser Trend hin zur Auflösung der Geschlechtskonnotation von Themen dient zwar nicht direkt der Beantwortung der Forschungsfragen, ist jedoch in Hinblick auf die Repräsentation der Interessen weiblicher und männlicher Heranwachsender ein wichtiger Aspekt und für weitere Forschungen von Interesse.

\section{Ausblick}

Bei der Befragung zeigte sich, dass die Experten die Geschlechterverteilung auf Grundlage ihrer subjektiven Beobachtung überwiegend richtig einschätzen. Es bedarf jedoch valider Informationen über die Geschlechterverteilung unter den Regisseur/-innen, damit die Mitglieder des Auswahl- und Jurygremiums diese in ihrer Entscheidung berücksichtigen können.

Die Bachelorthesis zeigt außerdem, dass die Evaluation von Filmwettbewerben notwendig ist, um die Förderung der jeweiligen Zielgruppe zu garantieren und zu optimieren. Nur dadurch können Defizite erkannt und dann kann ihnen entgegengewirkt werden. Die Kombination aus quantitativer Auswertung und qualitativen Interviews stellt sich dabei als adäquate Methode zur Untersuchung der Verteilung bestimmter soziokultureller Merkmale unter den Teilnehmenden und deren Ursachen dar. Dadurch kann ebenfalls überprüft werden, ob sich die Struktur der Teilnehmen- den innerhalb der Preisträger widerspiegelt oder ob es durch den Auswahlprozess zu einer Verschiebung kommt. Intern können Wettbewerbe damit das Erreichen ihrer spezifischen Ziele und die Einhaltung von Richtlinien überprüfen. Die Veröffentlichung von Statistiken über die Struktur der Teilnehmenden ist jedoch ebenso wichtig, damit die aktive Filmarbeit Förderpotenziale erkennen und in ihren Konzepten und Methoden berücksichtigen kann.

Den Ergebnissen der Arbeit folgend bedarf es mehr weiblicher Heranwachsender, die sich aktiv mit dem Medium Film beschäftigen. Um dieses Ziel zu erreichen, sind nicht primär geschlechtsspezifische Angebote nötig, sondern eine Förderung der in sich gegebenen Verschränkung von Medien- und Genderkompetenz. Dazu dürfen sich die (Medien-)Pädagogen nicht von Geschlechterzuschreibungen leiten lassen, sondern müssen den individuellen Interessen und Sichtweisen jedes und jeder Einzelnen offen begegnen. Damit die aktive Medienarbeit geschlechtsspezifische Fähigkeiten und Defizite in der Förderung von Medienkompetenz berücksichtigen kann, sind Studien über die unterschiedlichen Nutzungskompetenzen verschiedener Medien nötig.

Da lediglich ein kurzer Zeitraum eines einzelnen Filmwettbewerbs untersucht wurde, bleibt zu überprüfen, ob die Erkenntnis der Ungleichverteilung der Geschlechter unter den Teilnehmenden des DJVPs nur auf diesen Wettbewerb oder auch auf weitere zutrifft. Die für diesen Artikel zusammengefasste Bachelorthesis möchte Impulse zur Untersuchung des bisher weitestgehend unerforschten Gebiets der Filmwettbewerbe geben und kann als Grundlage dafür Verwendung finden.

\section{Anmerkung}

1 Die Arbeit wurde im WS 2013/14 erstellt und von Prof. Dr. Horst Niesyto und JanRené Schluchter (Abteilung Medienpädagogik) betreut.

\section{Literatur}

Barg, Werner/ Niesyto, Horst/ Schmolling, Jan (Hrsg.) (2006): Jugend: Film: Kultur. Grundlagen und Praxishilfen für die Filmbildung. München.

Buchen, Sylvia/ Straub, Ingo (2006): Die Bedeutung des Hacker-Topos für Hauptschüler in der Adoleszenz. In: Treibel, Annette/ Maier, Maja S./ Kommer, Sven/ Welzel, Manuela (Hrsg) (2006): Gender medienkompetent. Wiesbaden, S. 93-111. 
Butler, Judith (1991): Das Unbehagen der Geschlechter. Frankfurt a.M.

Decke-Cornill, Helene/ Luca, Renate (2010): Jugend - Film - Gender. Medienpädagogische, bildungstheoretische und didaktische Perspektiven. Hannover.

Lauffer, Jürgen/ Röllecke, Renate (Hrsg.) (2011): Gender und Medien. Schwerpunkt: Medienarbeit mit Jungen. Beiträge aus Forschung und Praxis. Prämierte Medienprojekte. München.

Luca, Renate/ Aufenanger, Stefan/ Grell, Petra (2007): Geschlechtersensible Medienkompetenzförderung. Mediennutzung und Medienkompetenz von Mädchen und Jungen sowie medienpädagogische Handlungsmöglichkeiten. Düsseldorf.

Stolzenburg, Elke (1993): Praktische Medienarbeit mit Mädchen. Zum Beispiel Video. In: Brenner, Gerd/ Niesyto, Horst (Hrsg.): Handlungsorientierte Medienarbeit: Video, Film, Ton, Foto. Weinheim/ München, S. 135-139.

Theunert, Helga (2005): Geschlecht und Medien. Der Umgang von Mädchen und Jungen mit Medien. In: Anfang, Günther (2005): Von Jungen, Mädchen und Medien: Theorie und Praxis einer geschlechtsbewussten und -sensiblen Medienarbeit. München, S. 11-22. 